\title{
RESISTENCIA ANTIMICROBIANA DE UROPATÓGENOS EN ADULTOS MAYORES DE UNA CLÍNICA PRIVADA DE LIMA, PERÚ
}

\author{
Jacqueline Miranda ${ }^{1, a}$, Joseph Pinto ${ }^{2, b}$, Margot Faustino ${ }^{3, a}$, Billy Sánchez-Jacinto ${ }^{3, a}$, Fabrizio Ramirez ${ }^{2, c}$
}

\section{RESUMEN}

Se describen los principales mecanismos de resistencia antimicrobiana mediante el sistema Vitek® 2 en uropatógenos aislados en adultos mayores de una clínica privada en Lima. Estudio descriptivo realizado entre enero de 2014 y octubre de 2016. Escherichia coli, Klebsiella pneumoniae y Proteus mirabilis obtuvieron una sensibilidad mayor a $80 \%$ frente a piperacilina/tazobactam, amikacina y carbapenems. Asimismo, 83,6\% de Escherichia coli fueron cepas sensibles a nitrofurantoina. El $41,7 \%$ de Escherichia coli, 50,9\% de Klebsiella pneumoniae y $50 \%$ de Proteus mirabilis fueron productoras de betalactamasas de espectro extendido. De igual modo, $60 \%$ de Pseudomonas aeruginosa fueron productoras de carbapenemasas. La modificación de sitio activo (PBP) y la inactivación enzimática por penicilinasas se presentaron en el 7,8\% de Enterococcus faecalis. La resistencia a aminoglicósidos se presentó en Escherichia coli $(27,1 \%)$, Klebsiella pneumoniae $(46,7 \%)$ y Proteus mirabilis $(84,6 \%)$ por la producción de enzimas modificadoras. Existe un incremento de la resistencia bacteriana en relación a la edad. La inactivación enzimática de betalactámicos y aminoglicósidos es el mecanismo de resistencia más frecuente.

Palabras clave: Farmacorresistencia Bacteriana ; Infecciones bacterianas; Geriatría; Perú (fuente: DeCS BIREME).

\section{ANTIMICROBIAL RESISTANCE OF UROPATHOGENS IN OLDER ADULTS IN A PRIVATE CLINIC IN LIMA, PERU}

\begin{abstract}
The main mechanisms of antimicrobial resistance are described using the Vitek® 2 system in uropathogens isolated in older adults from a private clinic in Lima. Descriptive study conducted between January 2014 and October 2016. Escherichia coli, Klebsiella pneumoniae, and Proteus mirabilis obtained a sensitivity greater than $80 \%$ against piperacillin/ tazobactam, amikacin, and carbapenems. Moreover, $83.6 \%$ of Escherichia coli were nitrofurantoin-sensitive strains. A $41.7 \%$ of Escherichia coli, $50.9 \%$ of Klebsiella pneumoniae, and $50 \%$ of Proteus mirabilis were producers of extended spectrum beta-lactamases (ESBL). Similarly, $60 \%$ of Pseudomonas aeruginosa were producers of carbapenemases. Active site modification (PBP) and enzymatic inactivation by penicillinases occurred in $7.8 \%$ of Enterococcus faecalis. Resistance to aminoglycosides was presented in Escherichia coli (27.1\%), Klebsiella pneumoniae (46.7\%), and Proteus mirabilis (84.6\%) for the production of modifier enzymes. There is an increase in bacterial resistance in relation to age. Enzymatic inactivation of beta-lactam antibiotics and aminoglycosides is the most common resistance mechanism.
\end{abstract}

Keywords: Bacterial Drug Resistance; Bacterial Infections; Geriatrics; Peru (source: MeSH NLM).

\footnotetext{
Clínica Centenario Peruano Japonesa. Lima, Perú.

Universidad Privada San Juan Bautista. Lima, Perú.

Universidad Peruana Cayetano Heredia. Lima, Perú.

Tecnólogo médico; ${ }^{\mathrm{b}}$ biólogo; ${ }^{\mathrm{c}}$ médico cirujano.

El artículo es parte de la tesis de pregrado de Jacqueline Miranda para obtener el grado de médico cirujano de la Universidad Privada San Juan Bautista. Recibido: 27/06/2018 Aprobado: 20/02/2019 En línea: 22/03/2019
} 


\section{INTRODUCCIÓN}

El descubrimiento de la penicilina marcó un hito en la historia de la medicina. Luego de casi nueve décadas, nos enfrentamos nuevamente a un futuro incierto, debido a que las bacterias han desarrollado mecanismos de adaptación frente a la acción de antimicrobianos, constituyendo un problema de salud pública que afecta en mayor grado a países en desarrollo como el Perú (1-3).

La tendencia a la aparición de cepas resistentes frente a varios grupos de antimicrobianos se ha visto impulsada por el uso inapropiado de antimicrobianos, la automedicación y el incumplimiento terapéutico ${ }^{(4,5)}$. Las consecuencias negativas del incremento de la resistencia bacteriana se ven expresadas en términos de morbilidad, mortalidad y gastos sanitarios derivados de la atención médica que podrían amenazar la sostenibilidad de los sistemas de salud ${ }^{(6)}$.

Un estudio realizado en Estados Unidos por Sánchez et al., sobre resistencia bacteriana de Escherichia coli en pacientes adultos y adultos mayores de servicios ambulatorios, concluye que la resistencia bacteriana en adultos mayores va en aumento. De igual modo, los resultados de una revisión sistemática reportan que existe un aumento en la prevalencia y en los tipos de $\beta$-lactamasas de espectro extendido producidas por aislamientos de Enterobacteriaceae en los hospitales de América Latina ${ }^{(7,8)}$.

La infección del tracto urinario ocupa el segundo lugar entre los procesos infecciosos, siendo la principal causa de bacteriemia y sepsis en adultos mayores. El tratamiento se basa en la etiología más probable y la sensibilidad esperada de los uropatógenos, descrita en mapas microbiológicos ${ }^{(9)}$.

A pesar de que, la asociación entre la edad avanzada y la resistencia bacteriana es conocida en la literatura, no se disponen de estudios locales que describan los mecanismos de resistencia en las cepas bacterianas que afectan a adultos mayores. Cabe destacar que, sólo existen estudios que se limitan a describir los patrones de susceptibilidad de los uropatógenos de la población mayor de 18 años, en la cual incluyen a los adultos mayores. Por tal motivo, es necesario evaluar la resistencia bacteriana en esta población a fin de optimizar sus alternativas terapéuticas.

El objetivo principal del presente estudio fue describir la resistencia bacteriana y sus mecanismos en uropatógenos aislados en pacientes geriátricos de una clínica privada en Lima, Perú.

\section{EL ESTUDIO}

Se realizó un estudio descriptivo y retrospectivo. La población de estudio estuvo conformada por adultos

\section{MENSAJES CLAVE}

Motivación para realizar el estudio. La asociación entre la edad avanzada y la resistencia bacteriana es conocida en la literatura; sin embargo, no se disponen de estudios locales que describan los mecanismos de resistencia en las cepas bacterianas que afectan a adultos mayores.

Principales hallazgos. Los mecanismos de resistencia bacteriana más frecuentes de uropatógenos fueron por inactivación enzimática y modificación de sitio activo.

Implicancias. Las consecuencias negativas del incremento de la resistencia bacteriana se ven expresadas en términos de morbilidad, mortalidad y gastos sanitarios que podrían amenazar la sostenibilidad del sistema de salud peruano.

mayores con urocultivos positivos, atendidos en la Clínica Centenario Peruano Japonesa (CCPJ) durante el periodo 2014-2016. La CCPJ es una institución de salud privada situada en Lima, Perú, categorizada como III-1, que atiende un aproximado de 800 pacientes por día, presta servicios de salud preventiva y asistencial a la comunidad en general y promueve y desarrolla actividades de investigación en el campo de las ciencias de la salud.

Se incluyeron urocultivos positivos de pacientes de 60 años o más que contaron con perfil de susceptibilidad. Se excluyeron los urocultivos que no contaron con los datos completos. Se analizó el total de urocultivos positivos que cumplieron con el criterio de inclusión durante el periodo 2014-2016.

La susceptibilidad antimicrobiana y los mecanismos de resistencia se determinaron por concentración mínima inhibitoria (CMI) por el método de microdilución en caldo y fueron procesadas en el analizador automatizado VITEK por colorimetría y turbidimetria. La interpretación se realizó de acuerdo a los puntos de corte del Clinical and Laboratory Standards Institute (CLSI) ${ }^{(10)}$. El control de calidad del analizador se realizó a través del programa de evaluación externa de la calidad de la Universidad Peruana Cayetano Heredia.

Los datos demográficos como edad, sexo y procedencia y los datos de laboratorio fueron extraídos del archivo de la clínica CCPJ y se ingresaron a una base de datos de Microsoft Excel 2010. Las variables categóricas fueron reportadas mediante frecuencias, mientras que, las variables continúas mediante promedio y desviación estándar (DE). Se realizó un análisis bivariado mediante la prueba de chi-cuadrado para evaluar asociación entre la sensibilidad y grupo etario. Se consideró un valor $p<0,05$ como estadísticamente significativo. El programa estadístico utilizado fue $\mathrm{R}$ Studio versión 1.0.44. 
El protocolo de este estudio fue aprobado por el Comité Institucional de Ética de la Clínica Centenario Peruano Japonesa, Lima-Perú.

\section{HALLAZGOS}

La edad promedio de los adultos mayores fue de 77 años (DE: 10,47) y según los resultados de urocultivo positivo se observó una mayor frecuencia de infección urinaria en mujeres $(81,7 \%)$ en relación a los varones (18,3\%).

El total de urocultivos positivos recolectados fue de 1389. La distribución de uropatógenos aislados según procedencia se describe en la Tabla 1 y la frecuencia de susceptibilidad antimicrobiana en la Tabla 2.

Se encontró asociación entre la edad y la susceptibilidad a antimicrobianos; nitrofurantoína, ceftriaxona, cefotaxima, ceftazidima, cefuroxima y amikacina $(p<0,05)$ fueron evaluados para Escherichia coli evidenciándose un descenso en la sensibilidad a medida que incrementa la edad del paciente (Tabla 3 ).

El mecanismo de resistencia más frecuente fue la producción de BLEE, que presentaron los aislamientos de enterobacterias aislados (Tabla 4). El mecanismo enzimático BLEE tipo CTX- M estuvo presente en el 65,2\% ( $n=279)$ de cepas de E. coli y en el 45,5\% $(n=25)$ de $K$. pneumoniae.
La resistencia a aminoglucósidos estuvo originada por enzimas modificadoras de la actividad (EMAG), la más frecuente en todas las cepas de bacilos resistentes a aminoglucósidos fue ANT(2")-I + AAC(3)-II, en E. coli $(27,7 \%)$, K. pneumoniae $(46,7 \%)$ y $P$. mirabilis $(84,6 \%)$. En Pseudomonas aeruginosa, el patrón de resistencia más frecuente fue GEN NET AMI TOB (92,3\%).

Los mecanismos de resistencia presentes en los aislamientos de $E$. faecalis frente a betalactámicos se originó por la modificación de proteínas fijadoras de penicilina (PBP) en el $7,8 \%(n=4)$ del total de aislamientos. Asimismo, la resistencia mediada por penicilinasa adquirida estuvo presente en la misma proporción.

Para la familia de aminoglucósidos, la resistencia de alto nivel (RAN) a gentamicina se presentó en $35,3 \% \quad(n=18)$ de $E$. faecalis, mientras que, la RAN a kanamicina y estreptomicina estuvo presente en $23,5 \%(n=12)$ y $19,6 \%$ $(n=10)$; respectivamente. Del total de 51 aislamientos de $E$. faecalis se aisló una cepa resistente a vancomicina $(2,0 \%)$ y dos cepas resistentes a linezolid (3,9\%).

\section{DISCUSIÓN}

En los últimos 25 años, no se han encontrado reportes locales de mapas microbiológicos de uropatógenos aislados en adultos mayores, siendo el grupo etario con mayor

Tabla 1. Distribución de uropatógenos según procedencia en la Clínica Centenario Peruano Japonesa, 2014-2016

\begin{tabular}{|c|c|c|c|c|c|}
\hline \multirow{2}{*}{ Microorganismo } & \multicolumn{2}{|c|}{ Hospitalización } & \multirow{2}{*}{ Microorganismo } & \multicolumn{2}{|c|}{ Ambulatorio } \\
\hline & $n=176$ & $\%$ & & $n=1213$ & $\%$ \\
\hline Escherichia coli & 99 & 56,3 & Escherichia coli & 927 & 76,4 \\
\hline Klebsiella pneumoniae & 15 & 8,5 & Klebsiella pneumoniae & 93 & 7,7 \\
\hline Enterococcus faecalis & 13 & 7,4 & Proteus mirabilis & 41 & 3,4 \\
\hline Pseudomonas aeruginosa & 12 & 6,8 & Enterococcus faecalis & 38 & 3,1 \\
\hline Candida albicans & 11 & 6,3 & Pseudomonas aeruginosa & 18 & 1,5 \\
\hline Enterobacter aerogenes & 4 & 2,3 & Candida albicans & 17 & 1,4 \\
\hline Proteus mirabilis & 3 & 1,7 & Streptococcus agalactiae & 10 & 0,8 \\
\hline Streptococcus agalactiae & 3 & 1,7 & Citrobacter freundii & 9 & 0,7 \\
\hline Staphylococcus epidermidis & 2 & 1,1 & Enterobacter aerogenes & 8 & 0,7 \\
\hline Citrobacter freundii & 2 & 1,1 & Staphylococcus epidermidis & 7 & 0,6 \\
\hline Candida glabrata & 2 & 1,1 & Morganella morganii & 7 & 0,6 \\
\hline Enterobacter cloacae & 2 & 1,1 & Acinetobacter baumannii & 5 & 0,4 \\
\hline Candida dublinensis & 1 & 0,6 & Staphylococcus saprophyticus & 5 & 0,4 \\
\hline Morganella morganii & 1 & 0,6 & Klebsiella oxytoca & 4 & 0,3 \\
\hline Cibrobacter koseri & 1 & 0,6 & Candida parapsilosis & 4 & 0,3 \\
\hline Burkholderia cepacia & 1 & 0,6 & Staphylococcus haemolyticus & 3 & 0,2 \\
\hline Candida tropicalis & 1 & 0,6 & Cibrobacter koseri & 2 & 0,2 \\
\hline \multirow[t]{2}{*}{ Staphylococcus saprophyticus } & 1 & 0,6 & Enterobacter cloacae & 2 & 0,2 \\
\hline & & & Otros & 6 & 0,6 \\
\hline
\end{tabular}


Tabla 2. Perfil de susceptibilidad de uropatógenos aislados en la Clínica Centenario Peruano Japonesa, 2014-2016

\begin{tabular}{|c|c|c|c|c|c|}
\hline \multirow{2}{*}{ Antimicrobianos } & $\begin{array}{c}\text { Escherichia } \\
\text { Coli }\end{array}$ & $\begin{array}{c}\text { Klebsiella } \\
\text { pneumoniae }\end{array}$ & $\begin{array}{l}\text { Proteus } \\
\text { mirabilis }\end{array}$ & $\begin{array}{c}\text { Pseudomonas } \\
\text { aeruginosa }\end{array}$ & $\begin{array}{c}\text { Enterococcus } \\
\text { faecalis }\end{array}$ \\
\hline & $\%$ & $\%$ & $\%$ & $\%$ & $\%$ \\
\hline \multicolumn{6}{|l|}{ Penicilinas } \\
\hline Ampicilina & 20,0 & $\mathrm{R}$ & 32,6 & $\mathrm{R}$ & 84,3 \\
\hline Ampicilina - Sulbactam & 32,9 & 36,3 & 67,4 & $\mathrm{R}$ & NA \\
\hline Pipercilina - Tazobactam & 93,0 & 80,6 & 100 & 44,4 & NA \\
\hline \multicolumn{6}{|l|}{ Cefalosporinas } \\
\hline Cefazolina & 54,0 & 44,9 & 44,2 & $\mathrm{R}$ & NA \\
\hline Cefuroxima & 55,1 & 46,6 & 44,2 & $\mathrm{R}$ & NA \\
\hline Ceftazidima & 56,5 & 49,1 & 45,5 & 36,7 & NA \\
\hline Ceftriaxona & 56,5 & 49,1 & 45,5 & $\mathrm{R}$ & NA \\
\hline Cefotaxima & 56,5 & 49,1 & 45,5 & $\mathrm{R}$ & NA \\
\hline Cefepime & 58,2 & 49,1 & 45,5 & 40,0 & NA \\
\hline \multicolumn{6}{|l|}{ Carbapenémicos } \\
\hline Meropenem & 99,9 & 99,1 & 95,5 & 40,0 & NA \\
\hline Ertapenem & 99,9 & 99,1 & 95,5 & & NA \\
\hline Imipenem & 99,9 & 99,1 & 97,7 & 40,0 & NA \\
\hline \multicolumn{6}{|l|}{ Aminoglucósidos } \\
\hline Gentamicina & 70,1 & 73,1 & 62,8 & 43,3 & 47,1 \\
\hline Amikacina & 94,6 & 96,2 & 100 & 40,0 & 70,6 \\
\hline \multicolumn{6}{|l|}{ Macrólidos } \\
\hline Eritromicina & NA & NA & NA & NA & 8,6 \\
\hline \multicolumn{6}{|l|}{ Fluoroquinolona } \\
\hline Ciprofloxacino & 36,8 & 48,6 & 40,9 & 36,7 & 74,0 \\
\hline \multicolumn{6}{|l|}{ Nitrofuranos } \\
\hline Nitrofurantoína & 83,6 & 37,1 & $\mathrm{R}$ & $\mathrm{R}$ & 91,8 \\
\hline
\end{tabular}

R: resistencia; NA: no aplica

riesgo de generar resistencia bacteriana. Por tal motivo, el presente estudio busca aumentar el conocimiento en este grupo poblacional.

Los establecimientos de salud suelen dividir a la población general en pediátricos y adultos, incluyendo a los adultos mayores en el segundo grupo. Sin embargo, se observa en este estudio que los pacientes adultos mayores presentan sensibilidades menores, planteando así, la necesidad de considerarlos en un grupo independiente en los futuros mapas microbiológicos, a fin de orientar mejor el tratamiento empírico en esta población ${ }^{(10,11)}$.

Las infecciones del tracto urinario son la segunda causa de procesos infecciosos en la patología humana, afectando sobre todo a las mujeres. A partir de los 50 años, la incidencia se equipara con el sexo masculino ${ }^{(12)}$. En nuestro estudio, a pesar de que, los urocultivos que se analizaron correspondían en su totalidad a pacientes mayores de 60 años, se observó una mayor frecuencia en mujeres $(81,7 \%)$ que en varones $(18,3 \%)$, resultados que fueron similares a los reportados en población adulta menor de 60 años en estudios previos ${ }^{(12,13)}$.

Nuestras cifras difieren de lo reportado por Aspichueta et al. ${ }^{(14)}$ quien encontró frecuencias cercanas entre el sexo masculino $(50,1 \%)$ y femenino $(49,9 \%)$ en un estudio realizado en pacientes geriátricos. Por otro lado, Villegas et al. reportó que las frecuencias en el género masculino tienden a crecer a partir de los 70 años ${ }^{(15)}$. En nuestro estudio, no se encontraron diferencias significativas en la distribución según genero de urocultivos positivos, a través de los subgrupos etarios.

Las enterobacterias fueron los agentes etiológicos más frecuentes concordando con lo descrito en las referencias ${ }^{(16,17)}$. En el análisis de uropatógenos según la procedencia del paciente, se observó que en el ambiente hospitalario, Escherichia coli representó el $56,3 \%$ en comparación al $76,4 \%$ encontrado en pacientes ambulatorios, siendo el agente etiológico más frecuente. Además, su proporción disminuyó en hospitalización, descenso que contribuyó al incremento en las proporciones de microorganismos como Enterococcus faecalis, Pseudomonas aeruginosa y Candida albicans, lo que complicaría el tratamiento por tratarse de bacterias que poseen elevada capacidad de adquirir resistencia. Estos hallazgos concuerdan con lo reportado por otros autores ${ }^{(18)}$.

En la población de estudio, la Candida albicans es uno de los seis uropatógenos más frecuentes, probablemente debido al estado de inmunosupresión característico de 
Tabla 3. Patrón de susceptibilidad antimicrobiana de Escherichia coli de acuerdo a subgrupos etarios en la Clínica Centenario Peruano Japonesa, 2014-2016.

\begin{tabular}{|c|c|c|c|c|}
\hline \multirow{3}{*}{ Antimicrobiano } & \multicolumn{3}{|c|}{ Sensibilidad por grupo etario (años) } & \multirow{3}{*}{ Valor de $p^{*}$} \\
\hline & $60-69$ & $70-79$ & $\geq 80$ & \\
\hline & $\mathrm{n}(\%)$ & $\mathrm{n}(\%)$ & n (\%) & \\
\hline Nitrofurantoina & $284(86,9)$ & $230(85,8)$ & $339(79,8)$ & 0,017 \\
\hline Ceftriaxona & $201(60,9)$ & $158(58,7)$ & $221(51,8)$ & 0,029 \\
\hline Cefotaxima & $201(60,9)$ & $158(58,7)$ & $221(51,8)$ & 0,029 \\
\hline Ceftazidima & $201(60,9)$ & $158(58,7)$ & $222(52,0)$ & 0,035 \\
\hline Amikacina & $318(97,8)$ & $245(94,2)$ & $383(92,3)$ & 0,038 \\
\hline Cefuroxima & $189(59,4)$ & $147(57,0)$ & $212(50,7)$ & 0,049 \\
\hline Cefepime & $206(62,4)$ & $159(59,1)$ & $232(54,3)$ & 0,076 \\
\hline Cefazolina & $187(57,7)$ & $146(55,7)$ & $212(50,1)$ & 0,096 \\
\hline TMP-SX & $136(41,2)$ & $103(38,3)$ & $148(35,0)$ & 0,214 \\
\hline Ciprofloxacino & $131(39,7)$ & $101(37,7)$ & $145(34,0)$ & 0,251 \\
\hline Ampicilina & $69(20,9)$ & $59(22,2)$ & $81(19,2)$ & 0,637 \\
\hline Gentamicina & $225(68,2)$ & $190(70,6)$ & $304(71,2)$ & 0,65 \\
\hline Ampicilina-Sulbactam & $111(33,7)$ & $90(34,0)$ & $132(31,5)$ & 0,737 \\
\hline PIperacilina-Tazobactam & $107(93,0)$ & $82(92,1)$ & $145(93,5)$ & 0,916 \\
\hline Meropenem & $330(100)$ & $269(100)$ & $426(99,8)$ & NE \\
\hline Ertapenem & $330(100)$ & $269(100)$ & $426(99,8)$ & NE \\
\hline Imipenem & $330(100)$ & $269(100)$ & $426(99,8)$ & NE \\
\hline
\end{tabular}

*Prueba chi-cuadrado

$\mathrm{NE}$ : no evaluado

la senescencia y la exposición a tratamientos de amplio espectro $^{(19)}$.

La proporción de producción de BLEE que presentaron los aislamientos de $E$ coli $(41,7 \%)$, K. pneumoniae $(50,9 \%)$ y Proteus mirabilis (50\%) fue superior a las reportadas en

Tabla 4. Frecuencia de producción de betalactamasas de espectro extendido en relación a la procedencia del paciente según tipo de servicio en la Clínica Centenario Peruano Japonesa, 2014-2016.

\begin{tabular}{|c|c|c|}
\hline \multirow{2}{*}{$\begin{array}{l}\text { Microorganismo/ } \\
\text { Mecanismo }\end{array}$} & \multirow{2}{*}{$\begin{array}{c}\text { Hospitalizados } \\
\mathrm{n}(\%)\end{array}$} & \multirow{2}{*}{$\begin{array}{c}\text { Ambulatorios } \\
\mathrm{n}(\%)\end{array}$} \\
\hline & & \\
\hline \multicolumn{3}{|l|}{ E. coli } \\
\hline BLEE + & $50(50,5)$ & $378(40,8)$ \\
\hline BLEE - & $49(49,5)$ & $549(59,2)$ \\
\hline \multicolumn{3}{|l|}{ K. pneumoniae } \\
\hline BLEE + & $5(33,3)$ & $50(53,8)$ \\
\hline BLEE - & $10(66,7)$ & $43(46,2)$ \\
\hline \multicolumn{3}{|l|}{$P$. aeruginosa } \\
\hline BLEE + & $2(16,7)$ & $0(0)$ \\
\hline BLEE - & $10(83,3)$ & $18(100)$ \\
\hline \multicolumn{3}{|l|}{ P. mirabilis } \\
\hline BLEE + & $2(66,7)$ & $20(48,8)$ \\
\hline BLEE - & $1(33,3)$ & $21(51,2)$ \\
\hline
\end{tabular}

Europa y Norteamérica, incluso mayores a las cifras para América Latina $(36,4 \%)^{(7,8)}$. Nuestra población presenta cifras mayores de producción de BLEE si las comparamos con lo reportado para una población menor de 60 años, que señalan a la edad $\geq 65$ años como un factor de riesgo para adquirir una infección urinaria por una cepa BLEE $(+)^{(20)}$.

La betalactamasa de espectro extendido tipo CTX-M fue la más frecuente en $E$. coli $(65,2 \%)$ mientras que, en K. pneumoniae y $P$. mirabilis representó el $45,5 \%$ del total de enzimas producidas. Nuestros resultados concuerdan con el cambio epidemiológico en relación a los tipos de BLEE a predomino de las cefotaximasas ${ }^{(21,22 .)}$.

La resistencia a aminoglucósidos estuvo medida por la producción de enzimas modificadoras, como mecanismo más frecuente y ampliamente investigado. La asociación enzimática más frecuente fue ANT(2")-I + AAC(3)-II en E. coli $(27,7 \%)$, K. pneumoniae $(46,7 \%)$ y $P$. mirabilis $(84,6 \%)$. En Pseudomonas aeruginosa, el patrón de resistencia más frecuente fue GEN NET AMI TOB (92,3\%). Así, conocer los mecanismos de resistencia de las bacterias ayudaría a que la modificación de los actuales aminoglucósidos se dirija a crear nuevos derivados que no sean vulnerables a la acción de esas enzimas.

El reducido número de aislamientos de Proteus mirabilis, Pseudomonas aeruginosa y Enterococcus faecalis y la baja frecuencia en infecciones del tracto urinario en los subgrupos 
etarios, no permitió valorar la existencia de la relación significativa entre la edad y los patrones de susceptibilidad. Sin embargo; consideramos que los resultados de este estudio son importantes, ya que plantean la necesidad de vigilar la resistencia bacteriana en pacientes geriátricos en nuestro país, así como, evaluar los mecanismos de resistencia de los uropatógenos. La modificación de antimicrobianos actuales y la producción de fármacos que esquiven los mecanismos de resistencia disminuirán la prevalencia de estos microrganismo y sus repercusiones en la salud pública.

En conclusión, los mecanismos más frecuentes de resistencia bacteriana en uropatógenos fueron inactivación enzimática (BLEE CTX-M y ANT(2")-I + AAC(3)-II) y modificación de sitio activo (PBP).

Contribuciones de autoría: JM y JP participaron en la concepción y diseño del estudio, JM en la recolección de datos, JM,JP, MF, FR, BS en el análisis e interpretación de datos, JP y BS con asesoría estadística, JM, MF, BS, JP y FR realizaron la revisión crítica del artículo. Todos los autores aprobaron la versión final del artículo y asumen la responsabilidad del contenido.

Fuentes de financiamiento: autofinanciado.

Conflicto de intereses: los autores declaran no tener ningún conflicto de interés.

\section{REFERENCIAS BIBLIOGRÁFICAS}

1. Wilson TS, Markland AD Urinary Tract Infections in Older Adults. In: Griebling T. (eds) Geriatric Urology. Springer, New York, 2014

2. Rowe TA, Juthani-Mehta M. Urinary tract infection in older adults. Aging health. 2013;9(5):10.2217/ahe.13.38.

3. Muvunyi C, Masaisa F, Bayingana C, Mutesa L, Musemakweri A, Muhirwa G, et al. Decreased Susceptibility to Commonly Used Antimicrobial Agents in Bacterial Pathogens Isolated from Urinary Tract Infections in Rwanda: Need for New Antimicrobial Guidelines. Am J Trop Med Hyg. 2011; 84(6):923-8.

4. Mihankhah A, Khoshbakht R, Raeisi M, Raeisi V. Prevalence and antibiotic resistance pattern of bacteria isolated from urinary tract infections in Northern Iran. J Res Med Sci. 2017;22:108. doi:10.4103/ jrms.JRMS_889_16.

5. Mandal J, Acharya NS, Buddhapriya D, Parija SC. Antibiotic resistance pattern among common bacterial uropathogens with a special reference to ciprofloxacin resistant Escherichia coli. Indian J Med Res. 2012;136(5):842-9.

6. TansarliS, Karageorgopoulos E, Kapaskelis A, Falagas E. Impact of antimicrobial multidrug resistance on inpatient care cost: An evaluation of the evidence. Expert Rev Anti Infect Ther. 2013;11(3):321-31.

7. Sanchez G, Spencer J, Adams E, Alexis M, Baird G. Escherichia coli antimicrobial resistance increased faster among geriatric outpatients compared with adult outpatients in the USA, 2000-10. J Antimicrob Chemother. 2013;68(8):1838-41.

8. Guzmán-Blanco M, Labarca A, Villegas V, Gotuzzo E. Extended spectrum $\beta$-lactamase producers among nosocomial Enterobacteriaceae in Latin America. Braz J Infect Dis. 2014;18(4):421-33.
9. Tumbarello M, Sali M, Trecarichi EM, Leone F, Rossi M, Fiori F. Bloodstream infections caused by extended-spectrumbetalactamase-producing Escherichia coli: risk factors for inadequate initial antimicrobial therapy. Antimicrob Agents Chemother. 2008;52:3244-3252.

10. Clinical and Laboratory Standards Institute. M100 - S25 Performance Standards for Antimicrobial Susceptibility Testing [Internet]. CLSI; 2015 [citado el 2 de febrero de 2019]. Disponible en: https://clsi.org/ media/1469/m100s27_sample.pdf

11. Ruiz de Castilla P. Infecciones urinarias en mayores de 60 años en hospitalizados del Centro Medico Naval "Cirujano Mayor Santiago Tavara”, [Tesis para optar por el título de médico cirujano]. Perú: Sistema de bibliotecas: Universidad Peruana Cayetano Heredia; 1992.

12. Castro R, Barreto A, Guzmán H, Ortega R, Benítez L. Patrones de resistencia antimicrobiana en uropatógenos gramnegativos aislados de pacientes ambulatorios y hospitalizados Cartagena, 2005-2008. Rev. salud pública. 2010;12(6):1010-1019

13. Villarroel E, Navarro P, Ramos R, Andrade E, Bolívar A, Marcano J. Escherichia coli identificadas en pacientes con infecciones urinarias: Sensibilidad antimicrobiana. Rev. Soc. Ven. Microbiol. 2002;22(1):18-21.

14. Aspichueta C, Sota M, Esteban V, Barrios J, Sánchez J. Urocultivos en un hospital general de pacientes $>65$ años durante el período 1997-2002 (septiembre) Hospital de Basurto. Bilbao. Enferm Infecc Microbiol Clin. 2003; 21 Supl 1:24.

15. Villegas M, Cortés C, Hidalgo C, Martín M, Casanova T, García I. Diferencias epidemiológicas en infecciones urinarias extrahospitalarias entre mayores y menores de 70 años. Enferm Infecc Microbiol Clin. 2003; 21 Supl 1:26.
16. Sánchez S, Reyes P, Bermudez D. Susceptibilidad microbiológica de los uropatógenos aislados en la comunidad en Colombia periodo 2009-2013. Rev.Medica.Sanitas. 2015; 18(2):54-64.

17. Nicolle LE. Update in adult urinary tract infection. Curr Infect Dis Rep. 2011; 13: 552-560.

18. Andreu A, Planells I, Grupo Cooperativo Español para el Estudio de la Sensibilidad Antimicrobiana de los Patógenos Urinarios. Etiología de la infección urinaria baja adquirida en la comunidad y resistencia de Escherichia coli a los antimicrobianos de primera línea. Estudio nacional multicéntrico. Med Clin. 2008;130(13):481-486.

19. Wilson ML, Gaido L. Laboratory diagnosis of urinary tract infections in adult patients. Clin Infec Dis. 2004; 38:11501158.

20. Ben-Ami R, Rodríguez-Baño J, Arslan $\mathrm{H}$, Pitout JD, Quentin C, Calbo ES, et al. A multinational survey of risk factors for infection with extended-spectrum betalactamase-producing Enterobacteriaceae. in nonhospitalized patients. Clin Infect Dis. 2009; 49(5):682-690

21. Martínez L, Calvo J. The growing problem of antibiotic resistance in clinically relevant Gram-negative bacteria: current situation. Enferm Infecc Microbiol Clin. 2010; Suppl 2:25-31.

22. Angel M, Ramón J, Martínez L, Rodríguez J, Pascual A. Extended-spectrum betalactamase producing Escherichia coli and Klebsiella pneumoniae in Spanish hospitals: 2nd multicenter study (GEIHBLEE project, 2006). Enferm Infecc Microbiol Clin. 2009; 27:503-510.

Correspondencia: Delia Margot Faustino Arias.

Correo electrónico:delia.faustino@upch.pe 